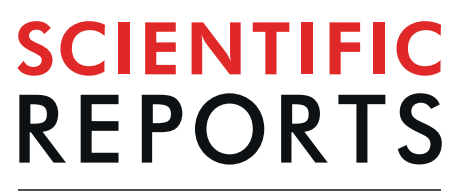

natureresearch

\title{
The depletion of ubiquilin in Drosophila melanogaster disturbs neurochemical regulation to drive activity and behavioral deficits
}

Salinee Jantrapirom $\mathbb{1}^{1,2}{ }^{2}$, Yosuke Enomoto ${ }^{3}$, Jirarat Karinchai ${ }^{4}$, Mizuki Yamaguchi², HidekiYoshida $^{2}$, Eiichiro Fukusaki ${ }^{3}$, Shuichi Shimma $\mathbb{B}^{3 *}$ \& Masamitsu Yamaguchi ${ }^{2 *}$

Drosophila melanogaster is a useful and highly tractable model organism for understanding the molecular mechanisms of human diseases. We previously characterized a new dUbqn knockdown model that induces learning-memory and locomotive deficits mediated by impaired proteostasis. Although proteinopathies are the main causes of neurodegenerative diseases, limited information is currently available on the relationship between proteostasis and neurodegenerative-related behavioral perturbations, such as locomotion, wakefulness, and sexual activities. Thus, the present study aimed to elucidate the mechanisms by which dUbqn depletion which is known to cause proteinopathies, affects neurodegenerative-related behavioral perturbations. Pan-neuronal dUbqn-depleted flies showed significantly reduced evening activity along with altered pre- and postsynaptic structural NMJ's proteins by attenuating signals of Bruchpilot puncta and GluRIIA clustering. In addition, the neurochemical profiles of GABA, glutamate, dopamine, and serotonin were disturbed and these changes also affected courtship behaviors in dUbqn-depleted flies. Collectively, these results extend our understanding on how dUbqn depletion affects neurochemical regulation to drive behavioral disturbances that are generally found in the early stage of neurodegenerative diseases. Moreover, the present study may contribute a novel finding to the design of new agents that prevent disease progression or even treat diseases related to neurodegeneration.

An effective regulation of proteostasis is a fundamental requirement for cells, particularly neurons, which are highly susceptible to proteotoxicity. Disruptions in protein turnover by stressors, gene mutations, or even a normal aging process have been shown to accelerate the development of proteinopathy-related disorders, such as Alzheimer's disease (AD), Parkinson's disease (PD), Amyotrophic Lateral Sclerosis (ALS), and Frontotemporal Dementia (FTD $)^{1-3}$. Neuronal-related symptoms, including motor, cognitive, and behavioral alterations, manifest in the early stages of neurodegenerative progression ${ }^{4,5}$. However, the mechanisms underlying these early symptoms have not yet been elucidated in detail.

Ubiquilins (UBQLNs), an important group of the proteins involved in proteostasis, has been extensively studied in a wide spectrum of neurodegenerative disorders. They are characterized as Ubiquitin-like (UbL)-Ubiquitin-associated (UbA) proteins that recognize and bind to ubiquitylated substrates in order to target them for degradation via proteasomes, endoplasmic reticulum-associated protein degradation (ERAD), and macroautophagy ${ }^{6-9}$. The five human $U B Q L N s$ homologues such as UBQLN1, UBQLN2, UBQLN3, UBQLN4 and $U B Q L N L$ have been established so far. However, only mutations in $U B Q L N 2$ and $U B Q L N 4$ strongly associate to ALS or ALS/FTD. Besides, UBQLNs also exhibit several distinctive activities, such as an actin and intermediate filament association, cell survival regulation by coordinating with protein-disulfide isomerase (PDI), and their emerging role in driving gene transcription by dUbqn-dHP1c-WOC-ROW complexes ${ }^{10-12}$.

\footnotetext{
${ }^{1}$ Department of Pharmacology, Faculty of Medicine, Chiang Mai University, Chiang Mai, 50200, Thailand. ${ }^{2}$ Department of Applied Biology, Kyoto Institute of Technology, Matsugasaki, Sakyo, Kyoto, 606-8585, Japan. ${ }^{3}$ Department of Biotechnology, Graduate School of Engineering, Osaka University, 2-1 Yamadaoka, Suita, Osaka, 565-0871, Japan. “Department of Biochemistry, Faculty of Medicine, Chiang Mai University, Chiang Mai, 50200, Thailand. *email: sshimma@bio.eng.osaka-u.ac.jp; myamaguc@kit.ac.jp
} 
Drosophila melanogaster is a useful and highly tractable model organism for studying and understanding the molecular mechanisms of human diseases due to biologically and physiologically highly conserved pathways between Drosophila and humans. Drosophila has a single homologue of UBQLN in its genome called $d U b q n$, which shows high similarity to human $U B Q L N 1,2$, and 4 in the major domains as well as functions ${ }^{13}$.

Bedford et al. discovered the regulatory function of UBQLNs in synapses to facilitate GABA-A receptor expression $^{14}$. Moreover, the dysregulation of dUbqn also contributes to defects in postsynaptic growth and synaptic proteostasis ${ }^{15}$. Although previous studies reported a role for UBQLNs in receptor regulation at synapses, its involvement in the control of neurochemical expression remains unclear. Dysfunctional UBQLNs with or without mutations cause ubiquitylated protein accumulation in the early stages of neurodegenerative diseases ${ }^{16}$. At the same stage, declines in motor-cognitive functions and behavioral changes, such as the loss of sexual abilities and sleep-awakening disturbances, are generally observed before the loss of affected neurons ${ }^{5,16-18}$. Therefore, the mechanisms by which dysfunctional UBQLNs affect neurochemical changes to induce motor, cognitive, and behavioral deficits are interesting to investigate in more detail.

Recently, our group took advantage of the novel mass spectrometry imaging (MSI) method to visualize neurochemicals inside the body of Drosophila ${ }^{19}$. In addition, with a newly established $d U b q n$ knockdown model we demonstrated that proteostasis impairments can drive learning-memory and locomotive deficits ${ }^{20}$. Thus, we intend to use the $d U b q n$ knockdown model to gain insights into the changes in more complex behaviors including copulation during proteostasis impairments through investigations on neurochemical regulation.

\section{Materials and Methods}

Drosophila handling and stocks. All flies were cultured at $25^{\circ} \mathrm{C}$ under humidity-controlled conditions. Standard Drosophila food contains $0.65 \%$ agar, $10 \%$ glucose, $4 \%$ dry yeast, $5 \%$ corn flour, and $3 \%$ rice powder ${ }^{19,20}$. Flies carrying UAS-dUbqnIR ${ }_{107-494}(106050)$ and $U A S-d U b q n I R_{471-824}(47447)$ and UAS-empty vector (60100) from the Vienna Drosophila Resource Center (VDRC), $w^{1118}$ (108479) from Kyoto stock center and those carrying elav-GAL4 (41500) and UAS-GFP-IR (41550) from the Bloomington Drosophila Stock Center (BDSC) were used in the present study. To minimize the effects of the genetic background, flies were backcrossed 6 times with the $w$ strain before experiments.

Drosophila activity assay. Newly eclosed adult male flies carrying UAS-dUbqnIR $107-494 /+; e l a v-G A L 4 /+$, +;elav-GAL4/UAS-dUbqnIR ${ }_{471-824}$ and UAS-GFP-IR/+;elav-GAL4/+ were selected under $\mathrm{CO}_{2}$ anesthesia. Three-day-old flies from each strain were individually placed in Drosophila activity monitoring tools (Trikinetics, Waltham, MA, USA) housed inside an incubator controlled at $25^{\circ} \mathrm{C}$. Fly activity was monitored for 7 days under a 12-h light-dark cycle ${ }^{21}$. The amount of activity by each fly was recorded every $30 \mathrm{~min}$.

Drosophila courtship assay. Pairs (virgin male and virgin female) of 3-day adult flies carrying

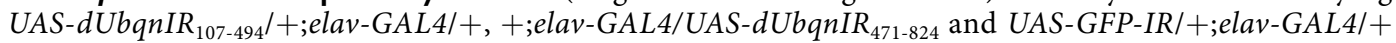
were selected under $\mathrm{CO}_{2}$ anesthesia. Briefly, flies were allowed to recover from anesthesia for at least $1 \mathrm{~h}$, and an aspirator was then used to gently transfer an individual male to the courtship chamber followed by a female ${ }^{22}$. Recordings of behavior were immediately started and conducted for at least 20 minutes.

Neuromuscular junctions (NMJs) visualization. Third instar larvae carrying $w^{1118}$, UAS-dUbqnIR ${ }_{107-494} /+;$ elav-GAL4/+, +;elav-GAL4/UAS-dUbqnIR ${ }_{471-824}$,Empty vector/+;elav-GAL4/+ and UAS-GFP-IR/+;elav-GAL4/+ were dissected in HL3 saline and fixed in Bouin's solution at $25^{\circ} \mathrm{C}$ for 30 minutes. Larval NMJs were incubated in $2 \%$ bovine serum albumin and $0.1 \%$ Triton-X 100 in PBS at $25^{\circ} \mathrm{C}$ for 30 minutes. Fluorescein isothiocyanate (FITC)-conjugated anti-horseradish peroxidase (HRP) IgG (1:1000) was used as the detection antibody ${ }^{20}$. Primary antibodies, such as mouse monoclonal anti-Disc large (Dlg) IgG (1:300), mouse monoclonal anti-Bruchpilot (Brp) IgG (1:50) and anti-GluRIIA IgG (1:80), were used to visualize active zones (AZ) under $4^{\circ} \mathrm{C}$ in a 16 -hour incubation. Samples were incubated with secondary antibodies labeled with Alexa 594 (1:400) before mounting with Vectashield (Vector Laboratories, Burlingame, CA, USA). Confocal microscopic images were taken with a confocal laser scanning microscope (Fluoview FV10i, Olympus, Tokyo, Japan) and processed with ImageJ software ${ }^{21}$.

Larvae tissue preparation. For sample treatment, third instar larvae at similar developmental stages (within 6 hours difference) were picked and dipped in $70 \%$ ethanol before embedding. Then, they were embedded in $10 \%$ gelatin on base-mold $(7 \mathrm{~mm} \times 7 \mathrm{~mm} \times 5 \mathrm{~mm} \text {; FALMA, Tokyo, Japan })^{23,24}$. Gelatin powder (Sigma-Aldrich, St. Louis, MO, USA) was dissolved in ultrapure water (Genpure UV-TOC xCAD PLUS, Thermo Fisher Scientific, Waltham, MA, USA) and mixed for $10 \mathrm{~min}$ at $42^{\circ} \mathrm{C}$, using a thermomixer (Eppendorf, Hamburg, Germany) until a homogeneous solution was obtained ${ }^{24}$. After aligning the larvae per their body axes in the embedding material, the molds were frozen rapidly in liquid nitrogen. The frozen blocks of gelatin with flies were placed in cryomicrotome (CM1950, Leica, Wetzlar, Germany) at $-20^{\circ} \mathrm{C}$ for 1 hour ${ }^{24}$. These blocks were then placed on a tissue-holder, fixed with an optimum cutting temperature polymer (Leica, Wetzlar, Germany), and sliced at $30-\mu \mathrm{m}$ thickness. The tissue sections were thaw-mounted onto Indium-tin-oxide (ITO)-coated glass slides (100 $\Omega / \mathrm{m}^{2}$ without anti-peeling coating, Matsunami Glass, Osaka, Japan) by warming them up by placing a finger on the posterior side of the glass slides ${ }^{24}$. The glass slides were then dried at $40^{\circ} \mathrm{C}$ for $5 \mathrm{~min}$ and placed in $50-\mathrm{mL}$ conical tubes containing silica gel ${ }^{24}$.

On tissue derivatization. 2,4-diphenyl-pyranylium tetrafluoroborate (DPP-TFB) (Sigma-Aldrich, St. Louis, MO, USA) was dissolved in methanol to prepare $10 \mathrm{mg} / \mathrm{mL}$ stock solutions ${ }^{23}$. DPP-TFB solutions used for derivatization contained $6 \mu \mathrm{L}$ of the stock solution, $69 \mu \mathrm{L}$ of $60 \%$ methanol, and $1 \mu \mathrm{L}$ of triethylamine. After 


\begin{tabular}{|l|l|l|l|l|l|}
\hline Analyte & $\begin{array}{l}\text { Precursor } \\
\text { ion }(\boldsymbol{m} / \boldsymbol{z})\end{array}$ & $\begin{array}{l}\text { Product } \\
\text { ion }(\boldsymbol{m} / \boldsymbol{z})\end{array}$ & $\begin{array}{l}\text { Q1 Pre } \\
\text { Bias }(\mathbf{V})\end{array}$ & $\begin{array}{l}\text { Collision } \\
\text { Energy }(\mathbf{e V})\end{array}$ & $\begin{array}{l}\text { Q3 Pre } \\
\text { Bias }(\mathrm{V})\end{array}$ \\
\hline Glutamate & 148.3 & 84.15 & -10 & -16 & -15 \\
\hline GABA & 103.9 & 87.2 & -12 & -14 & -15 \\
\hline Dopamine & 154.3 & 137.1 & -12 & -15 & -14 \\
\hline Serotonin & 177 & 160.1 & -21 & -13 & -16 \\
\hline Internal standard & 123 & 51 & -15 & -32 & -18 \\
\hline
\end{tabular}

Table 1. Parameters of MRM transition for neurochemicals and internal standard.

sectioning, on-tissue derivatization was performed with $50 \mu \mathrm{L}$ of DPP-TFB solution deposited onto each section manually using an airbrush (PS-270, GSI Creos, Tokyo, Japan $)^{23}$. Then the tissue sections were incubated for 90 minutes at $25^{\circ} \mathrm{C}$ to facilitate the derivatization reaction.

Matrix application. 2,5-dihydroxybenzoic acid (DHB) (Sigma-Aldrich, St. Louis, MO, USA) was applied to tissue sections via spray coating method ${ }^{24}$. DHB solution $(50 \mathrm{mg} / \mathrm{mL}$ dissolved in $50 \%$ methanol) was applied to each section using an airbrush (PS-270, GSI Creos, Tokyo, Japan $)^{24}$. The airbrush was maintained at $10 \mathrm{~cm}$ distal from glass slides and an approximately $50 \mu \mathrm{L}$ DHB solution was sprayed to each section and then completely dried. The spray-dry process was repeated multiple times ${ }^{24}$.

Mass spectrometry imaging (MSI) for neurochemicals. MSI was performed using an iMScope TRIO (Shimadzu, Kyoto, Japan). Both optical images and ion distribution were obtained within the same instrument under atmospheric pressure ${ }^{24}$. Nd:YAG laser $(\lambda=355 \mathrm{~nm}, 1 \mathrm{kHz})$ was used as the MALDI laser source, and laser irradiation was repeated 150 times on each data point with a laser power of 45.0 (arbitrary unit in iMScope TRIO $)^{24}$. The voltages of the sample stage and detector were $3.50 \mathrm{kV}$ and $2.10 \mathrm{kV}$, respectively ${ }^{24}$. A positive ion detection mode was used with an $\mathrm{m} / z$ range of 200-400 for detection of neurochemicals. Tandem mass spectrometry (MS/MS) analysis was carried out to visualize DPP-derivatized GABA, glutamate, dopamine, and serotonin with the selected precursor $m / z 318,362,368$, and 391. The ion peak in the product ion mass spectra provided the peak at $\mathrm{m} / z 232.11$, therefore the peak intensity of $\mathrm{m} / z 232.11$ was visualized for neurochemicals imaging. The IMAGEREVEAL (Shimadzu, Kyoto, Japan) was used for the data analyses.

Sample preparation for the Liquid chromatography-tandem mass spectrometry (LC-MS/MS) analysis. Five third instar larvae at similar developmental stages in 1.5-mL Eppendorf tubes were freeze-dried for $12 \mathrm{~h}$ using a freeze dryer (VD-800F, Taitec, Tokyo, Japan). Freeze-dried samples were milled using a ball mill (Retsch, Haan, Germany) at $20 \mathrm{~Hz}$ for $1 \mathrm{~min}$. Five hundred microliters of extraction solvent (methanol/water/ chloroform $=5: 2: 2 \mathrm{v} / \mathrm{v} / \mathrm{v} \%$ ) and $20 \mu \mathrm{L}$ of $1 \mathrm{mM} \mathrm{3,4-dihydroxybenzylamine} \mathrm{as} \mathrm{an} \mathrm{internal} \mathrm{standard} \mathrm{were} \mathrm{added}$ to each tube with the ball-milled sample. The sample was mixed using a vortex and sonicated for 1 minute. After sonication, the sample was centrifuged at $10000 \times g$ at $4{ }^{\circ} \mathrm{C}$ for 10 minutes. After centrifugation, $400 \mu \mathrm{L}$ of the supernatant was transferred to a new tube containing $150 \mu \mathrm{L}$ of water. Then the sample was mixed by vortexing and centrifuged at $10000 \times g$ at $4{ }^{\circ} \mathrm{C}$ for 10 minutes to separate the polar and non-polar phases. In the GABA, glutamate, and dopamine analysis, the upper polar phase was diluted by 10 -fold using water with $0.1 \%$ formic acid and syringe filtration (0.2- $\mu \mathrm{m}$ PTFE hydrophilic membrane, Merck) was performed. The filtered solution was transferred to a LC vial. In the serotonin analysis, $300 \mu \mathrm{L}$ of the upper polar phase was transferred via syringe filtration. The sample was then concentrated by centrifugation for 2 hours and freeze-dried for 12 hours. After dissolving in $30 \mu \mathrm{L}$ of $30 \%$ methanol, the sample was transferred to a $\mathrm{LC}$ vial.

LC-MS/MS analytical conditions. The LC-MS/MS analysis was performed using the Nexera UPLC System (Shimadzu, Kyoto, Japan) connected to LCMS-8050 (Shimadzu, Kyoto, Japan) with the ion source of electrospray ionization in the multiple reaction monitoring (MRM) mode ${ }^{24}$. Chromatographic separation was performed using a PFP column $(2.1 \times 100 \mathrm{~mm}, 3 \mu \mathrm{m}$, Shimadzu GLC, Tokyo, Japan $)$ at $40^{\circ} \mathrm{C}$ with an injection volume of $1 \mu \mathrm{L}$. The mobile phases were water with $0.1 \%$ formic acid (eluent $\mathrm{A}$ ) and acetonitrile with $0.1 \%$ formic acid (eluent B) with a gradient program: 0.01 minutes, $2 \% \mathrm{~B} ; 4.0$ minutes, $2 \% \mathrm{~B} ; 6.0$ minutes, $80 \% \mathrm{~B} ; 7.0$ minutes, $100 \% \mathrm{~B} ; 8.3 \mathrm{~min}$ utes, $100 \% \mathrm{~B} ; 8.31$ minutes, $2 \% \mathrm{~B}$; and 10 minutes, $2 \% \mathrm{~B}$. The flow rate was $0.3 \mathrm{~mL} /$ minute with a total run time of 10 minutes. The ion source was operated in the positive ion mode and interface temperatures were set as follows: nebulizer gas: $3.0 \mathrm{~L} /$ minute; heating gas: $10 \mathrm{~L} /$ minute; drying gas: $10 \mathrm{~L} /$ minute; interface temperature: $300^{\circ} \mathrm{C}$; desolvation line temperature: $250^{\circ} \mathrm{C}$; and heat block temperature: $400^{\circ} \mathrm{C}$. MRM conditions are summarized in Table 1. Data acquisition and processing were performed using LabSolutions (Shimadzu, Kyoto, Japan).

Data analysis. All statistical analyses were performed using GraphPad Prism version 6.02. The Mann-Whitney U test was used to assess the significance of differences between two independent groups ${ }^{20}$. The Kruskal-Wallis test followed by Dunnett's multiple comparison analysis was used to assess the significance of differences between three or more independent groups. All data were expressed as a mean \pm standard deviation (S.D.). $p<0.05$ was considered statistically significant. 


\section{Results}

Pan-neuronal dUbqn depletion decreases evening activity in D. melanogaster. We previously characterized $d U b q n$ RNAi flies and found that the knockdown of $d U b q n$ in all neurons caused an accumulation of ubiquitylated proteins and affected negative geotaxis in flies ${ }^{19,20}$. The proteostasis impairments caused by $d U b q n$ depletion appeared to negatively affect neuronal homeostasis and associated with neurodegeneration. Although a progressive reduction in the neuronal proteostasis capacity is known to generate a normal neuronal aging, it may result in an occurrence of neurodegenerative diseases. Some of the disease developing patients might suffer from a difficulty in sleeping which certainly affects their circadian rhythm ${ }^{25-27}$. Thus, we hypothesized that ubiquilins might be one of the key players in a circadian rhythm alteration and this relationship is worth to investigate.

Due to the fact that, Drosophila exhibit daily cycles in their behavioral rhythms that are governed by endogenous circadian rhythms ${ }^{28}$. Therefore, we examined whether the all-day activity or behavioral rhythms of Drosophila are disturbed by the knockdown of $d U b q n$. Flies carrying UAS- $d U b q n I R_{107-494} /+;$ elav-GAL4/+ (dUbqn depletion) and UAS-GFP-IR/+;elav-GAL4/+ (control), 3 days after eclosion were used to monitor daily activities by counting the number of times in 30 minutes that each individual fly crossed an infrared light beam. The $d U b q n$ RNAi lines with different targeted sequences were used to confirm and minimize the possibility of the off-target effects, as demonstrated previously in various assays ${ }^{19,20}$ and also in the present study (Figs. S1-S3). Both RNAi lines carrying elav $>d U b q n I R_{107-494}$ and elav $>d U b q n-I R_{471-824}$ similarly shortened the synapse branch length at NMJ (Fig. S1) and reduced the levels of the dUbqn protein and mRNA to the similar extent (Fig. S2). Control flies showed a regular pattern of activity based on peak activities twice a day between 6.30 to 10.00 am and between 9.00 to $10.00 \mathrm{pm}$, which are defined as the morning and evening peaks, respectively, and resting activities twice a day between $10.30 \mathrm{am}$ to $6.00 \mathrm{pm}$ and between $10.00 \mathrm{pm}$ to $6.00 \mathrm{am}$, which are defined as the midday siesta and nighttime sleep, respectively (Fig. 1A).

In comparisons with control flies, $d U b q n$-depleted flies showed a significant decrease in evening activity (evening peak) in the $1-5$ days of activity measurements (Fig. $1 \mathrm{~A}, \mathrm{~B}$ ), but only a slight reduction in morning activity (Fig. 1A,C). On days 3 and 5 , we observed a significant increase in activity in $d U b q n$-depleted flies during the resting period, such as the midday siesta, and activity at nighttime also appeared to increase (Fig. 1A,D,E). These results suggest that $d U b q n$-depleted flies exhibited decreased activity, particularly in the evening, which is consistent with our previous findings showing defects in the locomotive activity of $d U b q n$-depleted flies ${ }^{20}$. Another possibility to explain lower evening activity in $d U b q n$-deficient flies is that their higher activity during resting (the midday siesta) might affect their activity in the next period of time (evening); however, further studies are needed to clarify this issue.

dUbqn plays a role in regulating the presynaptic terminals of the Drosophila NMJ. AZ is an important structure for the exchange of chemicals at synapses in which the release of neurochemicals onto postsynaptic receptors is precisely controlled ${ }^{29,30}$. Alterations in AZs have been observed in various Drosophila disease models, particularly in neurological disorders, such as ALS, FTD, and ASD ${ }^{21,31,32}$. The immunostaining signals of Bruchpilot (Brp), one of the synaptic proteins located at the AZs of the Drosophila NMJs, were attenuated in the Drosophila model of ALS and Pitt-Hopkins syndrome ${ }^{33,34}$. Based on previous findings showing defects in the locomotive activity of $d U b q n$-depleted flies ${ }^{19,20}$ and the present results in which flies showed marked reductions in evening peak activity, we hypothesized that reductions in the locomotive activity of $d U b q n$-depleted flies may be caused by alterations in AZs at the presynaptic terminals of the NMJ.

The majority of adult fly motor neurons originally develop from larval motor neurons ${ }^{35}$. Therefore, we evaluated the expression of Brp by quantifying the density of Brp-positive puncta on NMJs. The Brp puncta were generally distributed throughout control NMJs (Fig. 2A-C), but were reduced in $d U b q n$-depleted NMJs (Fig. 2D-F). Due to the differences in the size and length of NMJs between group $\mathrm{s}^{20}$, we decided to quantify the number of Brp puncta by normalization to the related bouton area. We found that the number of Brp puncta was significantly lower in $d U b q n$-depleted NMJs than in control NMJs (Fig. 2G), suggesting that locomotive defects in $d U b q n$-depleted flies might be possibly caused by presynaptic alterations.

Theoretically, neurons compensate for changes in excitatory synaptic outputs to maintain a normal synaptic output $^{36}$. Reductions in Brp expression in the Drosophila NMJs may reflect a deficiency in the presynaptic output, and, thus, compensatory processes may take function to optimize a stability of neurotransmission by increasing the highly abundant glutamate receptor (GluR) in the postsynaptic area of the Drosophila NMJs ${ }^{37}$. Vice versa, when GluRIIA receptors are reduced, homeostasis retrospectively responds by increasing presynaptic glutamate release from $\mathrm{AZs}^{38,39}$. The distribution of GluR in the NMJ can be monitored by one of its subunits, GluRIIA.

Therefore, we examined the level of GluRIIA at NMJs in $d U b q n$-depleted larvae. The results revealed that postsynaptic GluRIIA signals normalized to the bouton area were markedly increased at the NMJs of $d U b q n$-depleted larvae (Fig. 3A-G). On the other hand, the GluRIIA cluster area was reduced in $d U b q n$-depleted larvae, possibly reflecting partial homeostatic compensation in $d U b q n$ depletion (Fig. 3A-F,H). Since the failure of neuronal compensatory mechanisms has been commonly observed in $\mathrm{PD}, \mathrm{AD}$, and other neurogenerative diseases, we speculated that the locomotive defects we found in $d U b q n$-depleted flies were possibly caused by presynaptic alterations together with a failure in synaptic compensation.

A number of neurochemicals were disturbed in whole bodies of dUbqn-depleted flies. Identifying the expression of neurochemicals is critical for interpreting neuronal and behavioral activities ${ }^{40-42}$. Based on the present results, we hypothesized that neurochemical dysregulation may be one of the factors affecting the changes observed in sleep-awake activity and locomotive ability in $d U b q n$-depleted flies. Thus, different kinds of neurochemicals, such as GABA, glutamate, dopamine, and serotonin, have been selected for detection using MSI to examine the distribution patterns of neurochemicals with the combination of LC-MS/MS for the relative quantitation of neurochemical levels. 
A
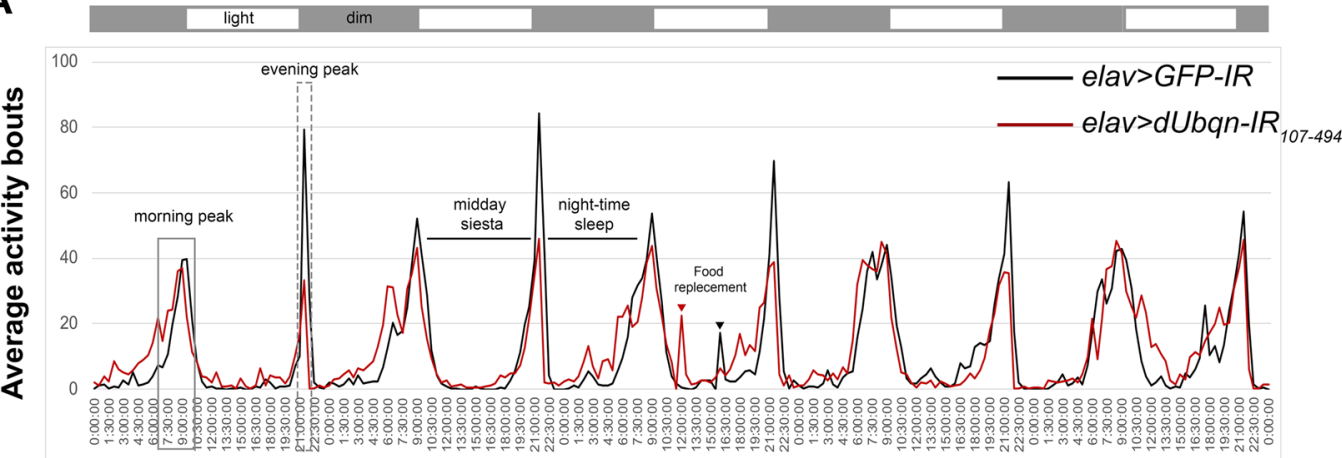

B

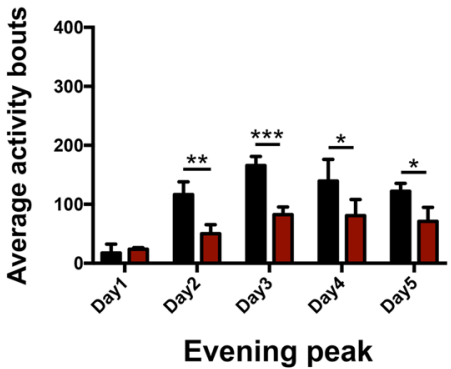

D

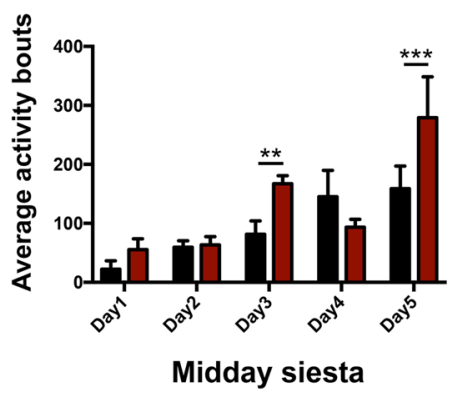

C

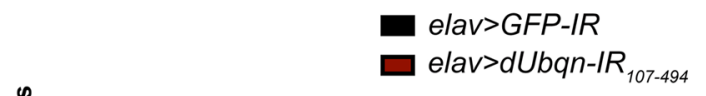

E

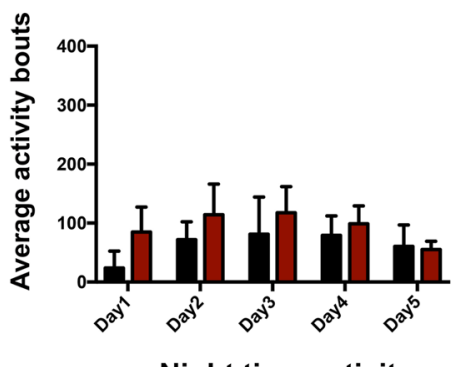

Figure 1. Pan-neuronal knockdown of $d U b q n$ reduced Drosophila evening activity. (A) The daytime and nighttime activity patterns of the following adult flies: elav > GFP-IR (black line, $w / Y$;UAS-GFP-IR/+;elavGAL4/+, $\mathrm{n}=30$ ) and elav $>d U b q n I R_{107-494}\left(\right.$ red line, $w / Y ; U A S-d U b q n I R_{107-494} /+;$ elav-GAL4/+, $\mathrm{n}=30$ ). Grey and white bars indicate 12 -h dark and 12-h light periods, respectively. All-day activity bouts are indicated, such as the evening period (Evening peak) (B), morning period (Morning peak) (C), afternoon period (Midday siesta) (D), and nighttime period (Nighttime activity) (E). The experiment was performed in triplicate. Error bars represent the standard deviation (S.D.) of data. $* \mathrm{p}<0.05, * * \mathrm{p}<0.01, * * * \mathrm{p}<0.001$.

Each of the neurochemicals showed a different pattern of distribution under control and $d U b q n$-depleted conditions. GABA and glutamate were detected alongside the larval bodies (Figs. 4A,B,D,E and S3A,B,D,E), whereas dopamine generally diffused throughout the body (Figs. 4G,H and S3G,H). Serotonin showed a pattern that differed from the others due to its dispersal near the internal organs of larvae (Figs. 4J,K and S3J,K). When we quantified the signal intensity of each neurochemical, the data revealed that $d U b q n$-depleted larvae (elav $\left.>d U b q n-I R_{107-494}\right)$ markedly showed an increase of GABA intensity (Fig. $4 \mathrm{C}$ ) whereas the other neurochemicals seemed to be declined as comparison to the control (Fig. 4F,I,L). Similarly, we have confirmed the significant decrease in glutamate and dopamine intensity with another $d U b q n$ RNAi line (elav $\left.>d U b q n-I R_{471-824}\right)$ along with a slightly increase and decrease of GABA and serotonin intensity, respectively compared to the control (Fig. S3A-L).

We have also quantified levels of each neurochemical by using LC-MS/MS. Under $d U b q n$-depleted conditions, the level of the inhibitory neurochemical, GABA was significantly higher (Fig. 5A) than under control conditions. The levels of excitatory neurochemicals, such as glutamate and dopamine, were significantly decreased (Fig. 5B,C), while those of serotonin were slightly decreased (Fig. 5D). These results suggested that the dysregulation of neurochemicals might be participated in order to reduce the activity in $d U b q n$-depleted flies. 

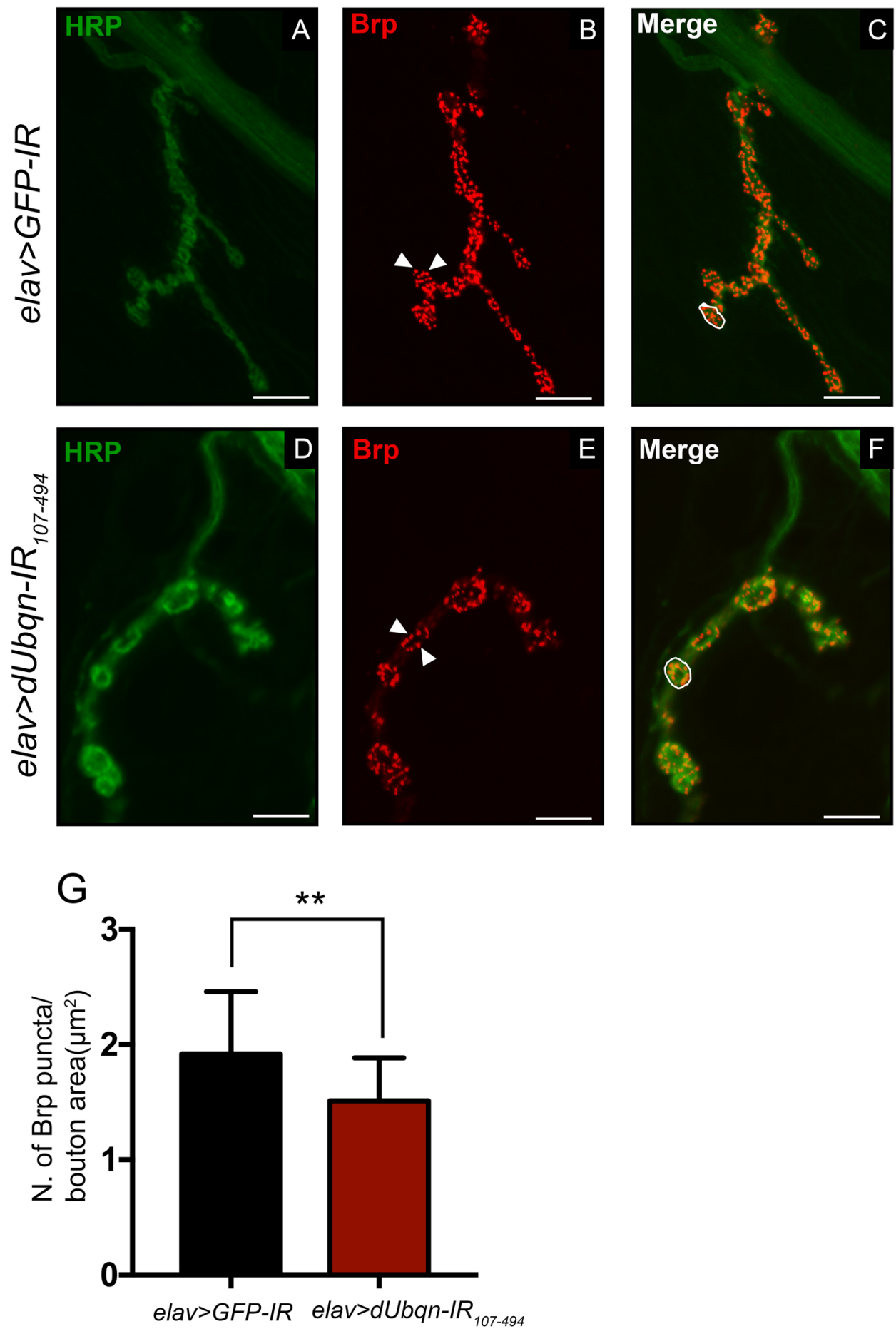

Figure 2. Pan-neuronal knockdown of $d U b q n$-altered Drosophila active zones. Confocal micrographs showing the terminal boutons of $4^{\text {th }} \mathrm{NMJs}$ from the third instar larvae of $(\mathbf{A}-\mathbf{C})$ elav $>G F P-I R(w / Y$; UAS-GFP$I R /+;$ elav-GAL4/+, $\mathrm{n}=12)$ and $(\mathbf{D}-\mathbf{F})$ elav $>d U b q n I R_{107-494}\left(w / Y ; U A S-d U b q n I R_{107-494} /+;\right.$ elav-GAL4/+, $\left.\mathrm{n}=12\right)$ immunolabeled with FITC-conjugated anti-HRP IgG (1:1000 dilution) (green) and anti-Brp IgG (1:50 dilution) followed by treatment with Alexa 594-conjugated anti-mouse IgG (1: 400 dilution) (red). Scale bar $=10 \mu \mathrm{m}$. (G) Quantification of the number of Brp puncta (examples are indicated by white arrowheads) per bouton area (examples are enclosed by white-line). The number of Brp puncta per bouton area was calculated by the total number of "Red spots" divided by the total area of boutons in each NMJ. Error bars represent the standard deviation (S.D.) of data. ${ }^{* *} \mathrm{p}<0.01$.

The perturbation of courtship behavior was observed in dUbqn-depleted flies. Drosophila has complex behaviors that are regulated by dopamine and serotonin such as feeding, locomotion, and courtship behaviors ${ }^{40,41,43,44}$. From LC-MS/MS data, we also found reductions in dopamine and serotonin levels under 

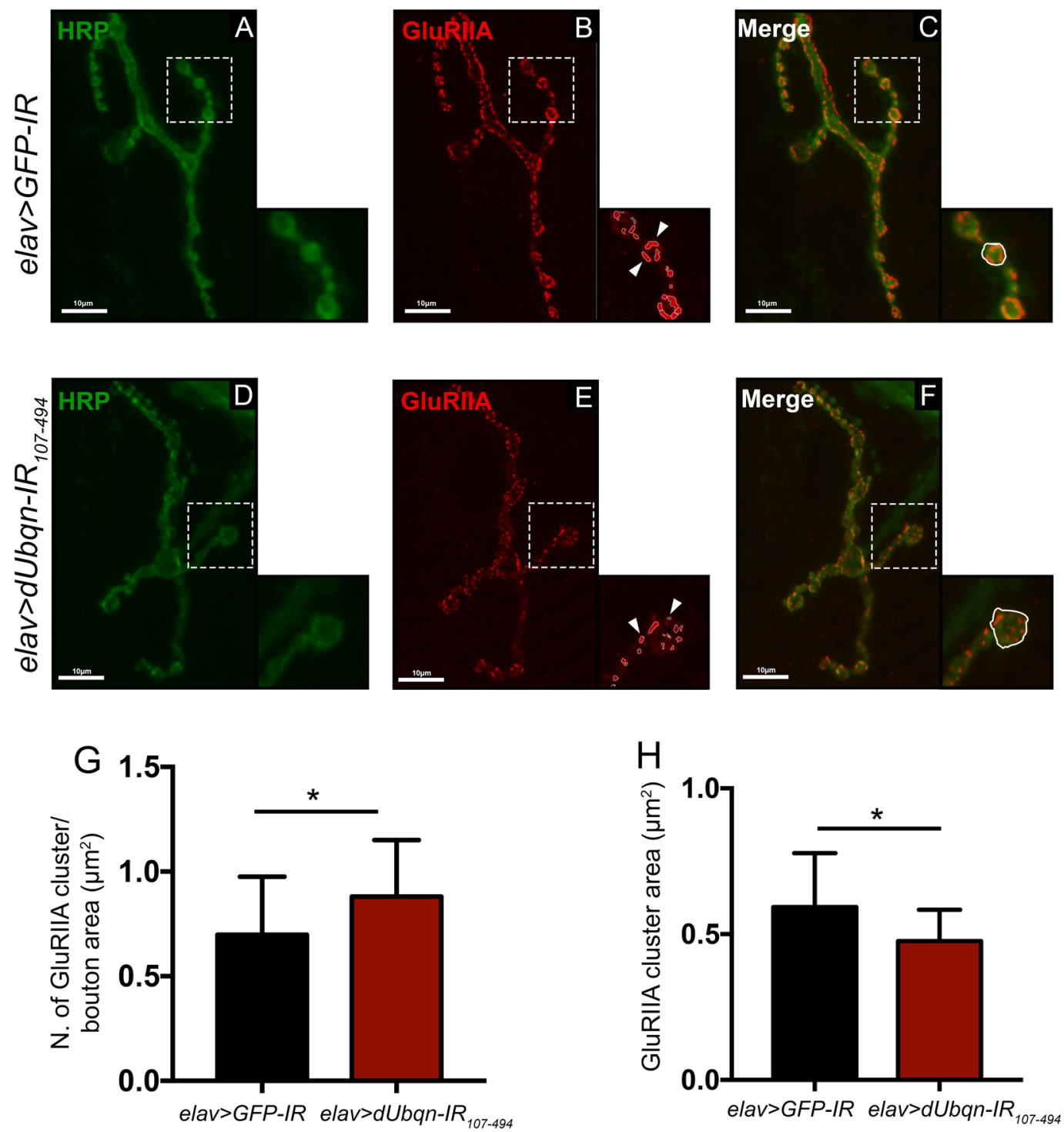

Figure 3. Pan-neuronal knockdown of $d U b q n$ altered Drosophila post-synaptic glutamate receptor clusters. Confocal micrographs showing the terminal boutons of $4^{\text {th }} \mathrm{NMJ}$ f from the third instar larvae of elav $>$ GFP-IR $(w / Y ; U A S-G F P-I R /+;$ elav-GAL4/+, $\mathrm{n}=12)(\mathbf{A}-\mathbf{C})$ and elav $>d U b q n I R_{107-494}\left(w / Y ; U A S-d U b q n I R_{107-494} /+;\right.$ elav$G A L 4 /+, \mathrm{n}=12)(\mathbf{D}-\mathbf{F})$ immunolabeled with FITC-conjugated anti-HRP IgG (1:1000 dilution) (green) and anti-GluRIIA IgG (1:80 dilution) followed by treatment with Alexa 594-conjugated anti-mouse IgG (1: 400 dilution) (red). Scale bar $=10 \mu \mathrm{m}$. (G) Quantification of the number of GluRIIA clusters (examples are indicated by white arrowheads) per bouton area (examples are enclosed by white-line). Number of GluRIIA cluster per bouton area was calculated by the total number of "Red spots" divided by the total area of boutons in each NMJ. (H) Quantification of the GluRIIA cluster area. GluRIIA cluster area was measure by an average area of each "Red spot". All results represent the mean \pm standard deviation (S.D.) of data. *p $<0.05$. The higher magnification image of indicated area of each panel is also shown.

$d U b q n$-depleted conditions. These results prompted us to examine whether $d U b q n$-depleted flies have dysfunctions in a fundamental behavior, such as their courtship behaviors.

Pairs of 3-day-old virgin flies were used to perform courtship behaviors. Most of the control and the dUbqn-depleted flies have completed copulation processes (Figs. 6B and S4A). During copulation latency, the male started orientation behaviors by turning toward and trying to catch the female. The male then tapped the female's body using his foreleg. The male made wing vibrations, called the "courtship song", extended his proboscis, and licked the female's genitalia. Courtship was considered to be successful following acceptance by the female to mounting and the male ejaculating his seminal fluid into the female (Fig. 6A). The time after mounting to separation was generally approximately $10-20$ minutes $^{22}$ and we observed approximately $12-17$ minutes of mounting in control and $d U b q n$-deficient pairs (Fig. 6D). An alteration of either copulation processes such as an extension of copulation latency or a shortening of copulation period might reveal some defects in courtship behaviors. The $d U b q n$-depleted pairs (elav $>d U b q n-I R_{107-494}$ ) had a longer copulation latency of nearly double 

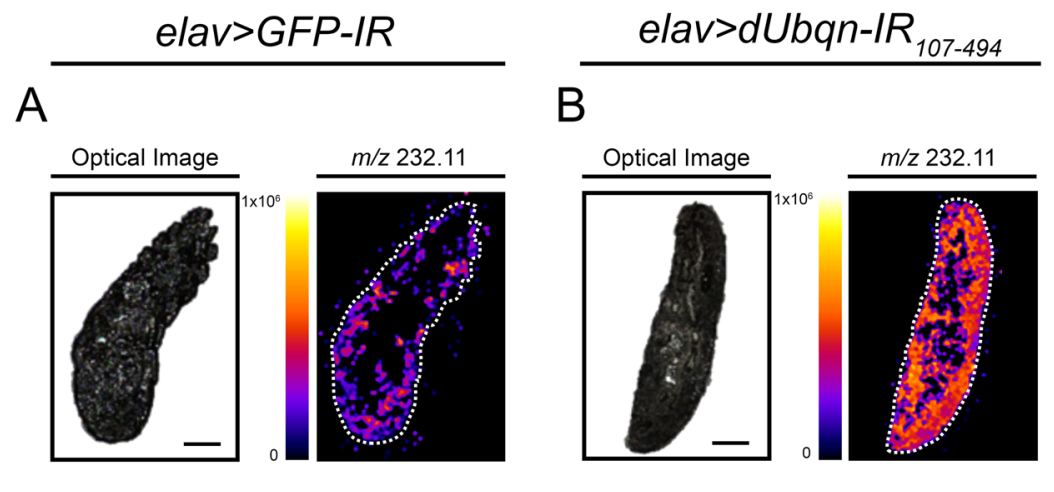

B

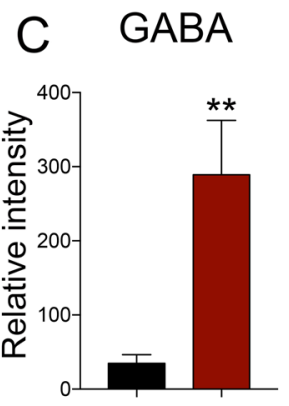

D

E

F Glutamate
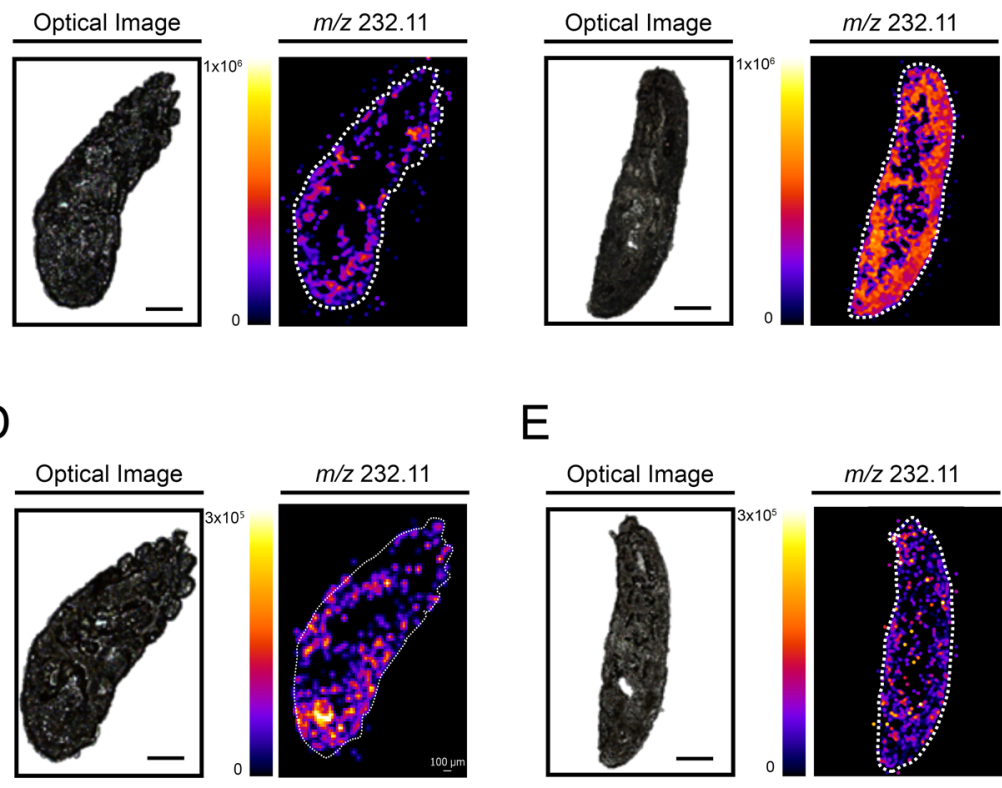

Optical Image
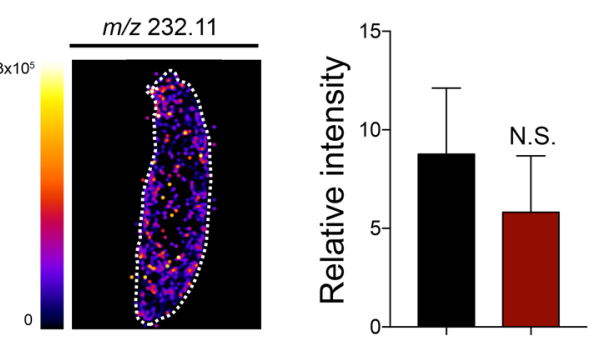

G

$\mathrm{H}$
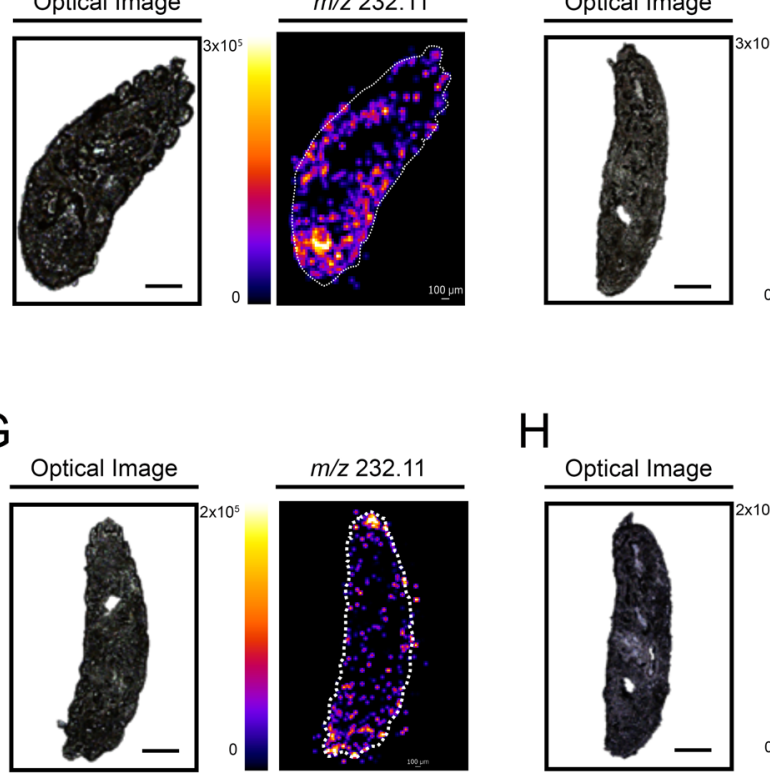

Dopamine
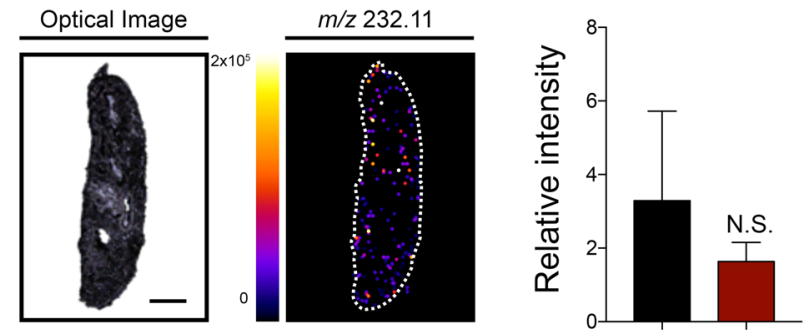

$J$

K

L Serotonin
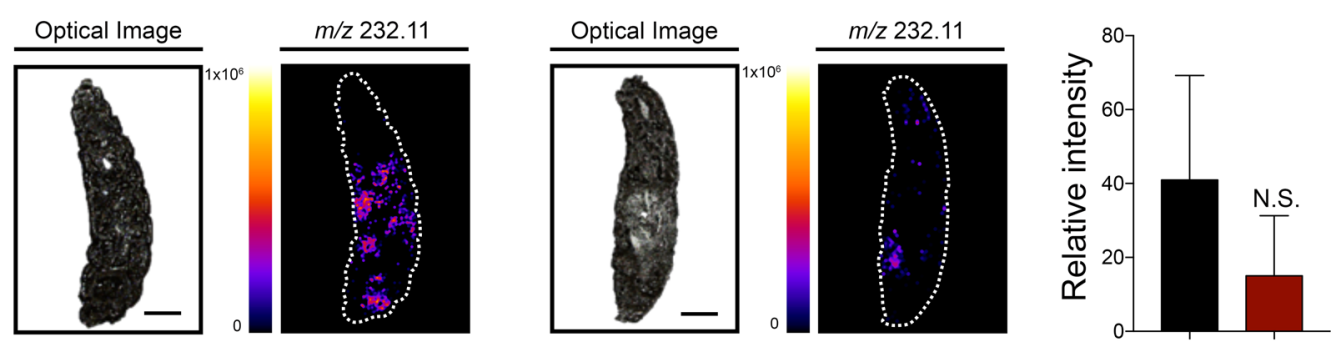

Figure 4. Pan-neuronal knockdown of $d U b q n$ disturbs neurochemical distribution. The distribution of GABA, glutamate, dopamine, and serotonin in the whole larval bodies of elav $>$ GFP-IR ( $w / Y$;UAS-GFP-IR/+;elav-GAL4/+, $\mathrm{n}=3)(\mathbf{A}, \mathbf{D}, \mathbf{G}, \mathbf{J})$ and elav $>U A S-d U b q n I R_{107-494}\left(w / Y ; U A S-d U b q n I R_{107-494} /+\right.$;elav-GAL4/+, $\left.\mathrm{n}=3\right)(\mathbf{B}, \mathbf{E}, \mathbf{H}, \mathbf{K})$. Optical images are shown on the left and the signal intensity map is shown on the right. White dashed lines indicate the area of larval bodies. Scale bar $=200 \mu \mathrm{m}$. Quantification of GABA (C), Glutamate (F), Dopamine (I) and Serotonin (L) intensity are shown. All results represent the mean \pm standard deviation (S.D.) of data. $* * p<0.01$ and N.S. $=$ not significant.

that of control pairs (Fig. 6C). It means that the affected flies required more time to recognize and respond to mating songs. In addition, a shortening of copulation period was also observed with another dUbqn RNAi line (elav $\left.>d U b q n-I R_{471-824}\right)$ (Fig. S4C). Cumulatively, our results suggested that an alteration in related neurochemical regulations such as dopamine and serotonin might be one of the causes of courtship behavioral defects in dUbqn-depleted flies. 
A

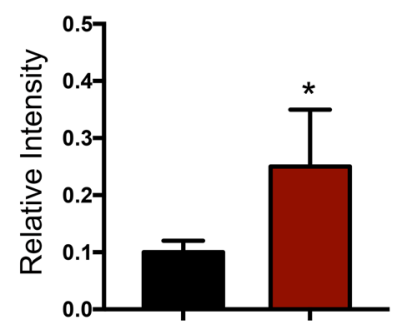

C

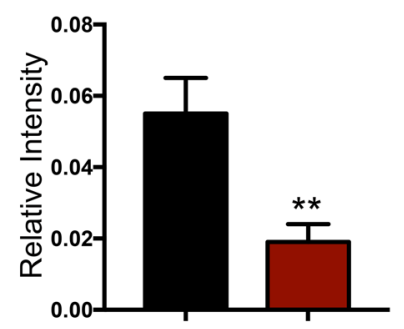

B

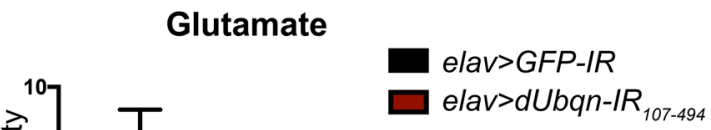

Figure 5. Pan-neuronal knockdown of $d U b q n$ changes neurochemical levels. The levels of GABA (A), glutamate (B), dopamine (C), and serotonin (D) in the whole larval bodies of elav > GFP-IR ( $w / Y$;UAS-GFP-IR/+;elav-GAL4 $/+, \mathrm{n}=3-5)$ and elav $>d U b q n I R_{107-494}\left(w / Y ; U A S-d U b q n I R_{107-494} /+; e l a v-G A L 4 /+, \mathrm{n}=3-5\right)$ are shown. All results represent the mean \pm standard deviation (S.D.). ${ }^{*} \mathrm{p}<0.05, * * \mathrm{p}<0.01$ and N.S. $=$ not significant.

A

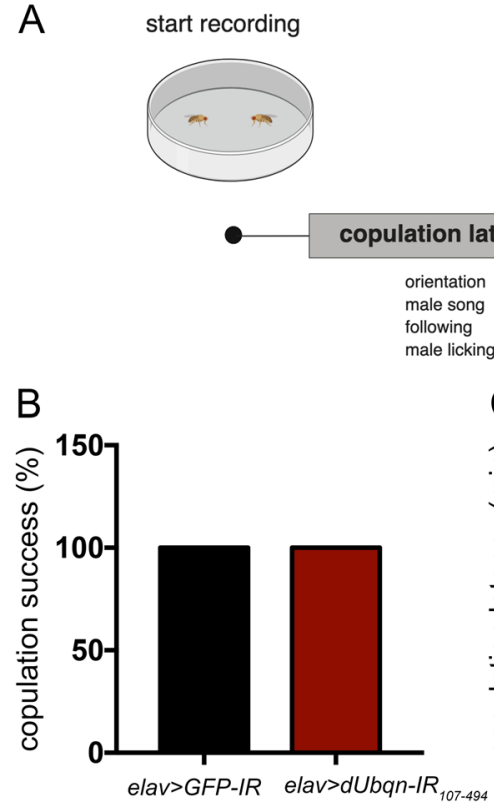

copulation

Serotonin
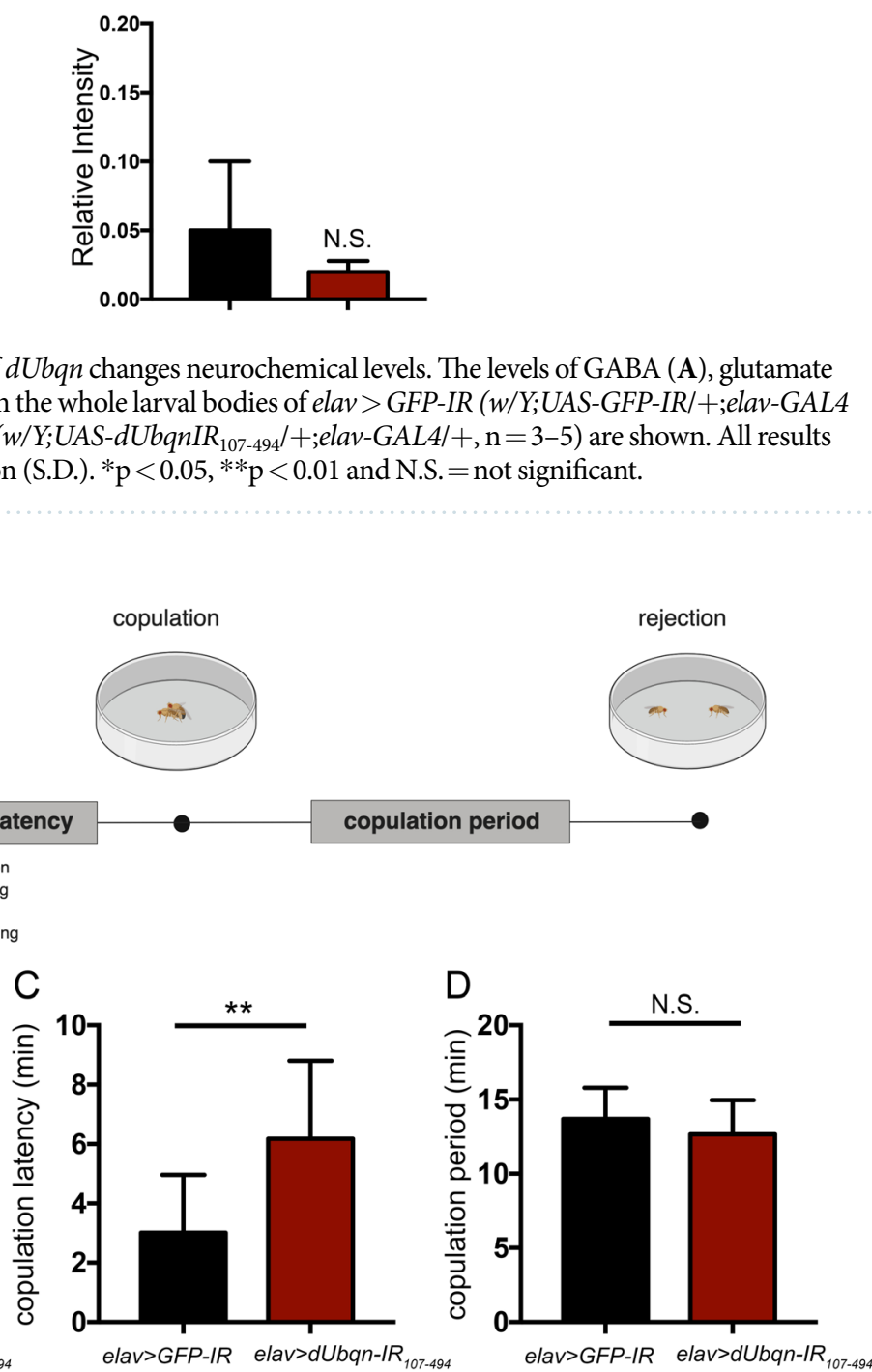

Figure 6. Pan-neuronal knockdown of $d U b q n$ causes defects in courtship behaviors. The algorithm of Drosophila courtship behaviors shows copulation latency and copulation period (A). The percentage of successful copulation (B), the copulation latency $(\mathbf{C})$, and copulation period (D) were compared in flies carrying elav $>$ GFP-IR $(w / Y$;UAS-GFP-IR/+;elav-GAL4/+, $\mathrm{n}=26)$ and elav $>d U b q n I R_{107-494}(w / Y$;UAS$d U b q n I R_{107-494} /+;$ elav-GAL4/+, $\mathrm{n}=26$ ). All results represent the mean \pm standard deviation (S.D.). $* * \mathrm{p}<0.01$.

\section{Discussion and Conclusion}

The present results demonstrated that pan-neuronal $d U b q n$ depletion, which is known to cause proteostasis impairments, reduced evening time activity and altered pre- and postsynaptic structural regulation in Drosophila NMJs. Moreover, the depletion of $d U b q n$, particularly in neurons, dysregulated neurochemicals, likely resulting in sexual behavioral alterations. 
We previously reported that $d U b q n$-depleted flies exhibit locomotive defects and aberrant NMJ macrostructures $^{20}$. The Drosophila activity monitoring assay, which follows the all-day activity of individual flies over many days, showed the daily rhythms of flies driven by endogenous circadian systems ${ }^{45}$. Consistent with our previous findings, we confirmed a reduction in locomotive activities of $d U b q n$-depleted flies, particularly in the evening peak, whereas normal flies performed peak activities during the same period. Moreover, $d U b q n$-depleted flies showed slightly increased activities during the resting period for many days, suggesting a disturbance in their circadian rhythms.

Brp is an important synaptic protein located in the AZs of neurotransmitter release sites ${ }^{46,47}$. Alterations in AZs have been found in various types of animal models related to neurological disorders, such as ALS, Pitt-Hopkins syndrome, and $\mathrm{ASD}^{21,33,34}$. Since $d U b q n$-depleted flies showed locomotive defects ${ }^{20}$, reductions in Brp may reflect synaptic defects in $d U b q n$-depleted flies that already exhibit difficulty with movement.

Homeostatic systems are considered to maintain the stability of neurotransmission. Synapses at the Drosophila NMJ are glutamatergic synapses that contain functional GluRIIA, GluRIIB, and other subunits ${ }^{38,48,49}$. Theoretically, the loss of both GluRIIA and GluRIIC subunits triggers a homeostatic increase in the quantal content to maintain sufficient neuronal activity ${ }^{38,39,48,50}$. On the other hand, the observed increase in GluR numbers in the present study may have been triggered by a compensatory mechanism to counteract reductions in presynaptic glutamate levels. Clustered GluRs are normally large and immobilized, whereas newly forming clusters are observed as the diffused form; therefore, reductions in the GluRIIA cluster area in $d U b q n$-depleted NMJs may reflect a sign of postsynaptic developmental defects that ultimately affect fly's movements.

Similar to humans, a number of neurochemicals are involved in the regulation of Drosophila activities and behaviors, such as glutamate, GABA, dopamine, and serotonin ${ }^{44,51,52}$. In the present study, we found a decrease in glutamate, an excitatory neurotransmitter, and increase in GABA, an inhibitory neurotransmitter, resulting in less activity, which is consistent with the results obtained in $d U b q n$-depleted flies. Considering the dopamine which is important for behavioral modulation in Drosophila especially an arousal ${ }^{53}$ and courtship behaviors ${ }^{54}$. Previous studies have reported that the dopamine-depleted female flies showed an increase in copulation latency whereas D1 dopamine receptor mutants in male flies found a delay in courtship initiation ${ }^{55,56}$. Moreover, we have found that $d U b q n$-depleted flies showed an alteration of dopamine and serotonin regulations that are confirmed the impact of the neurochemicals on sexual behaviors observed in our study. The sexual behavioral defects in humans might be caused by both mental and physical mechanisms. However, due to the limitation of the invertebrates. We cannot clearly justify whether the defects caused by mental or physical problems. Considering our experimental design, the courtship parameters that we have chosen such as the copulation latency and the copulation period, persuaded us to investigate the physical state rather than the mental state. However, the mammalian models are needed for further investigation.

Ubiquitylation is a critical mechanism controlling synaptic plasticity and architecture ${ }^{57,58}$, and its dysregulation has been implicated in numerous neurological disorders and psychiatric diseases ${ }^{59-61}$. Due to the roles of ubiquilins, which are closely related to ubiquitylation, further studies are warranted to clarify the mechanisms by which ubiquilin regulates synapses as well as neurochemicals. Despite the neurochemicals in both central and peripheral nervous system has been detected in our study, an exploration in the specific area is necessary and meaningful. An accumulation of the neurochemicals in some area outside brains might be caused by the specific release of the neurochemicals from the neurons in the peripheral nervous system. However, we cannot exclude the possibility that they are released in the process of preparing tissues, although the signals could be detected only inside larval bodies. Further analysis is necessary to understand the meaning of these observations.

The present study is the first to reveal the role of dUbqn in the regulation of synapses and neurochemicals, and impairments in its function may ultimately affect fundamental activities including locomotive and sexual activities. The $d U b q n$-deficient model with proteostasis impairments may be an important model for identifying early-stage markers of neurological disorders and developing promising therapies in the future.

\section{Data availability}

All of the original data are available on request.

Received: 2 July 2019; Accepted: 11 March 2020;

Published online: 30 March 2020

\section{References}

1. Pace, M. C. et al. Changes in proteome solubility indicate widespread proteostatic disruption in mouse models of neurodegenerative disease. Acta Neuropathol. 136, 919-938 (2018).

2. Kurtishi, A., Rosen, B., Patil, K. S., Alves, G. W. \& Møller, S. G. Cellular Proteostasis in Neurodegeneration. Mol. Neurobiol., 56, 3676-3689 (2018).

3. Hipp, M. S., Kasturi, P. \& Hartl, F. U. The proteostasis network and its decline in ageing. Nat. Rev. Mol. Cell Biol., 20, 421-435 (2019).

4. Seeley, W. W., Crawford, R. K., Zhou, J., Miller, B. L. \& Greicius, M. D. Neurodegenerative Diseases Target Large-Scale Human Brain Networks. Neuron 62, 42-52 (2009).

5. Levenson, R. W., Sturm, V. E. \& Haase, C. M. Emotional and behavioral symptoms in neurodegenerative disease: a model for studying the neural bases of psychopathology. Annu. Rev. Clin. Psychol. 10, 581-606 (2014).

6. Seok, K. H., Uehara, T., Tsuruma, K. \& Nomura, Y. Ubiquilin interacts with ubiquitylated proteins and proteasome through its ubiquitin-associated and ubiquitin-like domains. FEBS Lett. 566, 110-114 (2004).

7. Lim, P. J. et al. Ubiquilin and p97/VCP bind erasin, forming a complex involved in ERAD. J. Cell Biol. 187, 201-217 (2009).

8. Xie, Z. \& Klionsky, D. J. Autophagosome formation: core machinery and adaptations. Nat. Cell Biol. 9, 1102-1109 (2007).

9. N'Diaye, E. N. et al. PLIC proteins or ubiquilins regulate autophagy-dependent cell survival during nutrient starvation. EMBO Rep. 10, 173-179 (2009).

10. Wu, A. L., Wang, J., Zheleznyak, A. \& Brown, E. J. Ubiquitin-related proteins regulate interaction of vimentin intermediate filaments with the plasma membrane. Mol. Cell 4, 619-25 (1999). 
11. Ko, H. S., Uehara, T. \& Nomura, Y. Role of ubiquilin associated with protein-disulfide isomerase in the endoplasmic reticulum in stress-induced apoptotic cell death. J. Biol. Chem. 277, 35386-92 (2002).

12. Kessler, R. et al. dDsk2 regulates H2Bub1 and RNA polymerase II pausing at dHP1c complex target genes. Nat. Commun. 6, 7049 (2015).

13. Li, A. et al. Isolation and characterization of the Drosophila ubiquilin ortholog dUbqln: in vivo interaction with early-onset Alzheimer disease genes. Hum. Mol. Genet. 16, 2626-2639 (2007).

14. Bedford, F. K. et al. GABAA receptor cell surface number and subunit stability are regulated by the ubiquitin-like protein Plic-1. Nat. Neurosci. 4, 908-916 (2001).

15. Wang, C.-H. et al. USP5/Leon deubiquitinase confines postsynaptic growth by maintaining ubiquitin homeostasis through Ubiquilin. Elife 6 (2017).

16. Olson, E. J., Boeve, B. F. \& Silber, M. H. Rapid eye movement sleep behaviour disorder: demographic, clinical and laboratory findings in 93 cases. Brain 123, 331-339 (2000).

17. Sheridan, P. L. \& Hausdorff, J. M. The Role of Higher-Level Cognitive Function in Gait: Executive Dysfunction Contributes to Fall Risk in Alzheimer's Disease. Dement. Geriatr. Cogn. Disord. 24, 125-137 (2007).

18. van Iersel, M. B., Hoefsloot, W., Munneke, M., Bloem, B. R. \& Olde Rikkert, M. G. M. Systematic review of quantitative clinical gait analysis in patients with dementia. Z. Gerontol. Geriatr. 37, 27-32 (2004).

19. Jantrapirom, S., Lo Piccolo, L., Yoshida, H. \& Yamaguchi, M. Depletion of Ubiquilin induces an augmentation in soluble ubiquitinated Drosophila TDP-43 to drive neurotoxicity in the fly. Biochim. Biophys. Acta - Mol. Basis Dis., 1864, 3038-3049 (2018).

20. Jantrapirom, S., Lo Piccolo, L., Yoshida, H. \& Yamaguchi, M. A new Drosophila model of Ubiquilin knockdown shows the effect of impaired proteostasis on locomotive and learning abilities. Exp. Cell Res. 362, 461-471 (2018).

21. Ueoka, I. et al. Novel Drosophila model for psychiatric disorders including autism spectrum disorder by targeting of ATP-binding cassette protein A. Exp. Neurol. 300, 51-59 (2018).

22. Ejima, A. \& Griffith, L. C. Measurement of Courtship Behavior in Drosophila melanogaster. CSH Protoc. 2007, pdb.prot4847 (2007).

23. Enomoto, Y., Nt An, P., Yamaguchi, M., Fukusaki, E. \& Shimma, S. Mass Spectrometric Imaging of GABA in the Drosophila melanogaster; Adult Head. Anal. Sci. 34, 1055-1059 (2018).

24. Ohtsu, S., Yamaguchi, M., Nishiwaki, H., Fukusaki, E. \& Shimma, S. Development of a Visualization Method for Imidacloprid in;Drosophila melanogaster via; Imaging Mass Spectrometry. Anal. Sci. 34, 991-996 (2018).

25. Ning, S. \& Jorfi, M. Beyond the sleep-amyloid interactions in Alzheimer's disease pathogenesis. J. Neurophysiol., jn.00118.2019, 122, $1-4(2019)$.

26. Leng, Y., Musiek, E. S., Hu, K., Cappuccio, F. P. \& Yaffe, K. Association between circadian rhythms and neurodegenerative diseases. Lancet Neurol. 18, 307-318 (2019).

27. Minakawa, E. N., Wada, K. \& Nagai, Y. Sleep Disturbance as a Potential Modifiable Risk Factor for Alzheimer’s Disease. Int. J. Mol. Sci. 20 (2019).

28. Pfeiffenberger, C., Lear, B. C., Keegan, K. P. \& Allada, R. Locomotor Activity Level Monitoring Using the Drosophila Activity Monitoring (DAM) System. Cold Spring Harb. Protoc., 2010, pdb.prot5518-pdb.prot5518 (2010).

29. Hallermann, S., Heckmann, M. \& Kittel, R. J. Mechanisms of short-term plasticity at neuromuscular active zones of Drosophila. HFSP J. 4, 72-84 (2010).

30. Smith, R. \& Taylor, J. P. Dissection and Imaging of Active Zones in the Drosophila; Neuromuscular Junction. J. Vis. Exp., 50, 2676 (2011).

31. Perry, S., Han, Y., Das, A. \& Dickman, D. Homeostatic plasticity can be induced and expressed to restore synaptic strength at neuromuscular junctions undergoing ALS-related degeneration. Hum. Mol. Genet. 26, 4153-4167 (2017).

32. Schoen, M. et al. Super-Resolution Microscopy Reveals Presynaptic Localization of the ALS/FTD Related Protein FUS in Hippocampal Neurons. Front. Cell. Neurosci. 9, 496 (2015).

33. Ratnaparkhi, A., Lawless, G. M., Schweizer, F. E., Golshani, P. \& Jackson, G. R. A Drosophila model of ALS: human ALS-associated mutation in VAP33A suggests a dominant negative mechanism. Plos One 3, e2334 (2008).

34. Zweier, C. et al. CNTNAP2 and NRXN1 Are Mutated in Autosomal-Recessive Pitt-Hopkins-like Mental Retardation and Determine the Level of a Common Synaptic Protein in Drosophila. Am. J. Hum. Genet. 85, 655-666 (2009).

35. Landgraf, M. \& Thor, S. Development of Drosophila motoneurons: Specification and morphology. Seminars in Cell and Developmental Biology 17, 3-11 (2006).

36. Frank, C. A. Homeostatic plasticity at the Drosophila neuromuscular junction. Neuropharmacology 78, 63-74 (2014).

37. Collins, C. A. \& DiAntonio, A. Synaptic development: insights from Drosophila. Curr. Opin. Neurobiol. 17, 35-42 (2007).

38. Petersen, S. A., Fetter, R. D., Noordermeer, J. N., Goodman, C. S. \& DiAntonio, A. Genetic analysis of glutamate receptors in drosophila reveals a retrograde signal regulating presynaptic transmitter release. Neuron 19, 1237-1248 (1997).

39. DiAntonio, A., Petersen, S. A., Heckmann, M. \& Goodman, C. S. Glutamate receptor expression regulates quantal size and quantal content at the Drosophila neuromuscular junction. J. Neurosci. 19, 3023-32 (1999).

40. Araujo, S. M. et al. Chronic unpredictable mild stress-induced depressive-like behavior and dysregulation of brain levels of biogenic amines in Drosophila melanogaster. Behav. Brain Res. 351, 104-113 (2018).

41. Monier, M., Nöbel, S., Danchin, E. \& Isabel, G. Dopamine and Serotonin Are Both Required for Mate-Copying in Drosophila melanogaster. Front. Behav. Neurosci. 12, 334 (2019).

42. Yamada, D. et al. GABAergic Local Interneurons Shape Female Fruit Fly Response to Mating Songs. J. Neurosci. 38, 4329-4347 (2018).

43. Das, S. et al. Electrical synapses mediate synergism between pheromone and food odors in Drosophila melanogaster. Proc. Natl. Acad. Sci. USA 114, E9962-E9971 (2017).

44. Martin, C. A. \& Krantz, D. E. Drosophila melanogaster as a genetic model system to study neurotransmitter transporters. Neurochem. Int. 73, 71-88 (2014)

45. Chiu, J. C., Low, K. H., Pike, D. H., Yildirim, E. \& Edery, I. Assaying locomotor activity to study circadian rhythms and sleep parameters in Drosophila. J. Vis. Exp., 43, 2157 (2010).

46. Weyhersmuller, A., Hallermann, S., Wagner, N. \& Eilers, J. Rapid Active Zone Remodeling during Synaptic Plasticity. J. Neurosci. 31, 6041-6052 (2011).

47. Wagh, D. A. et al. Bruchpilot, a protein with homology to ELKS/CAST, is required for structural integrity and function of synaptic active zones in Drosophila. Neuron 49, 833-844 (2006).

48. Marrus, S. B., Portman, S. L., Allen, M. J., Moffat, K. G. \& DiAntonio, A. Differential Localization of Glutamate Receptor Subunits at the Drosophila Neuromuscular Junction. J. Neurosci. 24, 1406-1415 (2004).

49. Schuster, C. M. et al. Molecular cloning of an invertebrate glutamate receptor subunit expressed in Drosophila muscle. Science (80-). 254, 112-114 (1991).

50. Brusich, D. J., Spring, A. M. \& Frank, C. A. A single-cross, RNA interference-based genetic tool for examining the long-term maintenance of homeostatic plasticity. Front. Cell. Neurosci. 9, 107 (2015).

51. Nässel, D. R. \& Winther, Å. M. E. Drosophila neuropeptides in regulation of physiology and behavior. Prog. Neurobiol. 92, 42-104 (2010).

52. Nässel, D. R. Substrates for Neuronal Cotransmission With Neuropeptides and Small Molecule Neurotransmitters in Drosophila. Front. Cell. Neurosci. 12, 83 (2018). 
53. Andretic, R., van Swinderen, B. \& Greenspan, R. J. Dopaminergic Modulation of Arousal in Drosophila. Curr. Biol. 15, 1165-1175 (2005).

54. Liu, T. et al. Increased Dopamine Level Enhances Male-Male Courtship in Drosophila. J. Neurosci. 28, 5539-5546 (2008).

55. Neckameyer, W. S. Dopamine and mushroom bodies in Drosophila: experience-dependent and -independent aspects of sexual behavior. Learn. Mem. 5, 157-65 (1998).

56. Lim, J. et al. The mushroom body D1 dopamine receptor controls innate courtship drive. Genes, Brain Behav. 17, 158-167 (2018).

57. Hegde, A. N. The ubiquitin-proteasome pathway and synaptic plasticity. Learn. Mem. 17, 314-27 (2010).

58. Cline, H. Synaptic plasticity: importance of proteasome-mediated protein turnover. Curr. Biol. 13, R514-6 (2003).

59. Tai, H.-C. \& Schuman, E. M. Ubiquitin, the proteasome and protein degradation in neuronal function and dysfunction. Nat. Rev. Neurosci. 9, 826-838 (2008).

60. Lehman, N. L. The ubiquitin proteasome system in neuropathology. Acta Neuropathol. 118, 329 (2009).

61. Mabb, A. M. \& Ehlers, M. D. Ubiquitination in Postsynaptic Function and Plasticity. Annu. Rev. Cell Dev. Biol. 26, 179-210 (2010).

\section{Acknowledgements}

We thank Dr. Masatoshi Tomaru for his advice regarding the courtship behavior assay. We also thank the Bloomington Drosophila Stock Center and Vienna Drosophila Genetic Resource Center for the fly lines. This study was partially supported by a Grant-in-Aid from the JSPS Core-to-Core Program B, Asia-Africa Science Platforms, JSPS KAKENHI Grant Number JP19K06659, Kyoto Institute of Technology (KIT) Advance Insect Research Promotion Center (AIRPC) Grant Number 2019001 and the Faculty of Medicine Research Fund Grant Number $107 / 2562$ to S.J.

\section{Author contributions}

S.J., S.S., E.F., H.Y. and Ma.Y. conceptualized and designed the study. S.J., Y.E. and J.K. performed experiments and analyzed data. S.J., Y.E. and Ma.Y. wrote the manuscript. Ma.Y., H.Y., and S.S. contributed to resources and edited the manuscript. Mi.Y. contributed to the revise experiments. All authors reviewed and approved the manuscript.

\section{Competing interests}

The authors declare no competing interests.

\section{Additional information}

Supplementary information is available for this paper at https://doi.org/10.1038/s41598-020-62520-y.

Correspondence and requests for materials should be addressed to S.S. or M.Y.

Reprints and permissions information is available at www.nature.com/reprints.

Publisher's note Springer Nature remains neutral with regard to jurisdictional claims in published maps and institutional affiliations.

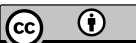

Open Access This article is licensed under a Creative Commons Attribution 4.0 International License, which permits use, sharing, adaptation, distribution and reproduction in any medium or format, as long as you give appropriate credit to the original author(s) and the source, provide a link to the Creative Commons license, and indicate if changes were made. The images or other third party material in this article are included in the article's Creative Commons license, unless indicated otherwise in a credit line to the material. If material is not included in the article's Creative Commons license and your intended use is not permitted by statutory regulation or exceeds the permitted use, you will need to obtain permission directly from the copyright holder. To view a copy of this license, visit http://creativecommons.org/licenses/by/4.0/.

(C) The Author(s) 2020 